\title{
The role of outpatient visit after operative treatment of ankle fractures
}

\section{Ovaska, Mikko}

2016-11

Ovaska, M , Nuutinen , T , Madanat , R, Makinen, T J \& Söderlund , T 2016 , ' The role of outpatient visit after operative treatment of ankle fractures ' , Injury, vol. 47 , no. 11 , pp.

2575-2578 . https://doi.org/10.1016/j.injury.2016.09.008

http://hdl.handle.net/10138/229654

https://doi.org/10.1016/j.injury.2016.09.008

publishedVersion

Downloaded from Helda, University of Helsinki institutional repository.

This is an electronic reprint of the original article.

This reprint may differ from the original in pagination and typographic detail.

Please cite the original version. 


\title{
The role of outpatient visit after operative treatment of ankle fractures
}

\author{
Mikko T. Ovaska, MD, PhD*, Timo Nuutinen, BM, Rami Madanat, MD, PhD, \\ Tatu J. Mäkinen, MD, PhD, Tim Söderlund, MD, PhD \\ Department of Orthopaedics and Traumatology, Helsinki University Hospital, Helsinki, Finland
}

\section{A R T I C L E I N F O}

\section{Keywords:}

Ankle fracture

Complication

Control visit

Radiograph

Infection

\begin{abstract}
A B S T R A C T
It is a common practice that patients have a scheduled follow-up visit with radiographs following ankle fracture surgery. The aim of this study was to evaluate whether an early outpatient visit ( $<3$ weeks) after ankle fracture surgery resulted in a change in patient management. For this study, 878 consecutive operatively treated ankle fracture patients with an early outpatient clinical-radiological visit were reviewed. The outcome measure was a change in treatment plan defined as any procedure, medication, or surgical intervention that is not typically implemented during the uncomplicated healing process of an acute fracture.

A change in treatment plan was observed in $9.8 \%$ of operatively treated ankle fracture patients. The mean age of the patients was 48 years and the mean follow-up time was 64 months. Of the changes in treatment plan, $91 \%$ were exclusively due to clinical findings such as infection. Only three of 878 patients required a change in their treatment plan based merely on the findings of the radiographs taken at the outpatient visit. Only $37 \%$ of the patients requiring a change in their postoperative management had solicited an unanticipated visit before the scheduled outpatient visit due to clinical problems such as infection or a cast-related issue.

Our study showed that every tenth operatively treated ankle fracture patient requires a change in their treatment plan due to a clinical problem such as infection or a cast-related issue. Although at hospital discharge all patients are provided with written instructions on where to contact if problems related to the operated ankle emerge, only one third of the patients are aware of the clinically alarming symptoms and seek care when problems present. Our findings do not support obtaining routine radiographs at the early outpatient visit in an ankle fracture patient without clinical signs of a complication.
\end{abstract}

(C) 2016 Published by Elsevier Ltd.

\section{Introduction}

Ankle fractures represent approximately $10 \%$ of all fractures and are among the most frequently surgically treated fractures [1,2]. Surgical treatment of ankle fractures may be accompanied by several complications $[3,4]$, and the most frequently encountered complications are related to wound healing and surgical-site infections $[1,4,5]$. Although the incidence of malreduction observed in postoperative radiographs leading to early reoperation is low [6], it is a common practice that patients have a scheduled follow-up visit to an outpatient clinic together with radiographs following acute fracture surgery [7].

Recent studies have questioned the justification of routine radiographs obtained at the early outpatient visit $[3,7]$, as the

\footnotetext{
* Corresponding author at: Department of Orthopaedics and Traumatology, Helsinki University Hospital, Topeliuksenkatu 5, 00260, Helsinki, Finland.

E-mail address: mikko.ovaska@hus.fi (M.T. Ovaska).
}

majority of these radiographs do not result in a change in patient management [7]. However, due to the high number of postoperative complications observed in ankle fracture patients, radiographs may be still indicated if the clinical picture necessitates them [3]. A recent study evaluating acute fractures in various anatomic locations showed that only a few patients require a deviation from the standard postoperative care at the first outpatient visit, and that the deviation is not based on the radiographs but rather on patient history and physical examination [7].

The first purpose of this study was to evaluate whether an early outpatient visit after ankle fracture surgery resulted in a change in patient management. The second goal was to assess how often the radiographs or the physical examination at the first outpatient visit after ankle fracture surgery resulted in a change in patient management. We hypothesized that the radiographs would not result in a change in patient management. However, the early outpatient visit might reveal problems related to wound healing in 
patients with ankle fracture surgery, and thus could not be totally abandoned.

\section{Materials and methods}

We performed a chart review of 878 consecutive operatively treated ankle fracture patients with an early ( $<3$ weeks) outpatient clinical-radiological visit at a level-I trauma centre from January 1st 2010 through December 31st 2011. Our hospital is located in an urban area with a catchment population of 1.9 million persons. All patients who had undergone operative treatment for an ankle fracture were identified by querying the hospital surgical procedure database for diagnoses coded using the International Classification of Diseases, Tenth Revision (ICD-10) as fibular fracture (S82.4), medial malleolar fracture (S82.5), lateral malleolar fracture (S82.6), bimalleolar or trimalleolar fracture (S82.8), and with the procedure code for internal fixation of an ankle fracture. Approval from our institutional review board was obtained prior to the beginning of the study. Eligible operations were restricted to those performed primarily at our institution and in patients 16 years of age or older and all fractures were definitively treated with open reduction and internal fixation (ORIF). Altogether, 61 surgeons performed all the ankle fracture operations during the 2-year study period.

A standardized operative and postoperative protocol was used during the study period. ORIF was performed based on AOprinciples and a tourniquet was applied depending on personal preferences of the treating surgeon. The wound was closed in three layers (peroneal fascia, subcutaneous layer, skin). Postoperatively, a cast was applied to all patients either in the operating room or during the following postoperative days and postoperative radiographs were obtained. Before hospital discharge all patients were provided with written instructions on where to contact if problems related to the operated ankle emerged, and an early scheduled outpatient visit within 3 weeks after surgery was appointed. Sutures or staples were removed and radiographs (anteroposterior, mortise, lateral) were taken at the early outpatient visit from all patients. After the first visit the patients were allowed to begin active ankle range of motion exercises. Full weight bearing was allowed at four weeks. At six weeks the patients had another outpatient visit, radiographs were taken, and the cast was removed.

Medical, operative, and radiological records of all 878 patients were reviewed in order to identify various characteristics of the patients and their injuries as well as possible changes in patient management. We collected the demographic data of the patients and the fracture type (Danis-Weber classification; uni-, bi-, or trimalleolar fracture; fracture-dislocation; open fracture). The medical and microbiological records were reviewed for recorded signs and symptoms for surgical site infection. We classified infections as deep when all three of the following criteria were met at the same time: clinical signs of a surgical site infection (redness, swelling, drainage, or dehiscence), positive bacterial cultures taken from the wound, and osteosynthesis material visible or palpable in the wound [8]. The remaining infections were considered as superficial.

Data concerning postoperative treatment plan were obtained by reviewing the physician's dictated clinic notes and radiographs from the first postoperative visit. Changes in normal postoperative management were defined as any procedure, medication, or surgical intervention that is not typically implemented during the uncomplicated healing process of an acute ankle fracture. These changes include local wound treatment or debridement, application of negative-pressure wound therapy, prescription of oral antibiotics, administration of intravenous antibiotics, unexpected changes in standard weight-bearing instructions, unexpected remodeling of the cast, removal of hardware excluding elective scheduled syndesmotic screw removal, and loss of fracture reduction requiring reoperation. We collected the number of patients who required a change in their treatment plan due to the findings of the first postoperative visit, and the causes for the change were divided into 1) clinical, 2) clinical and radiological, 3) radiological only. The exact causes for the change in the treatment plan were analyzed. The number of patients who required an unanticipated visit already before the scheduled outpatient visit was recorded and the causes for that visit were noted. The mean follow-up time was 64 months.

\section{Results}

The mean age of the patients was 48 years (range 16-91 years) and $60 \%$ of the patients were women. The basic fracture characteristics are presented in Table 1. Altogether, 86 of 878 (9.8\%) patients required a change in treatment plan due to the findings of the first outpatient visit including 18 patients requiring surgical treatment in the operating theatre (Fig. 1). Of these 86 patients, $32(37 \%)$ patients had solicited an unanticipated control visit prior to the scheduled outpatient visit due to emerging clinical problems related to the ankle fracture surgery (Fig. 2). Therefore, of the 86 patients requiring a change in postoperative management, $54(6.2 \%)$ patients required a change merely based on the findings of the scheduled early outpatient visit (Fig. 1).

Interestingly, $91 \%$ of the causes for a change in treatment plan were exclusively due to clinical findings such as infection or castrelated problems (Figs. 1 and 2). Only three $(0.3 \%)$ patients of cohort of 878 ankle fracture patients required a change in their treatment plan based merely on the findings of the radiographs taken at the early outpatient visit; in one of these three patients the cause was an undiagnosed medial malleolar fracture leading changes in weight-bearing. In the two other patients a partial loss of reduction of the medial malleolus without a need for reoperation was observed resulting in further restrictions in weight-bearing.

At the first outpatient visit, 78 (8.9\%) of 878 patients showed signs of infection. According to our previously defined criteria, $28 \%$ (22 of 78) of these infections were classified as deep and the remaining $72 \%$ were classified superficial infections. By the time of last control visit, 70 (8\%) of 878 patients had eventually been treated for problems related to deep surgical site infection.

Table 1

Fracture characteristics of the patients with an early outpatient visit following ankle fracture surgery $(n=878)$.

\begin{tabular}{ll}
\hline Characteristics & $\mathrm{n}(\%)$ \\
\hline Weber classification & \\
A & $11(1)$ \\
B & $605(69)$ \\
C & $234(27)$ \\
Other $^{\text {a }}$ & $28(3)$ \\
Fracture type & \\
$\quad$ Unimalleolar & \\
Bimalleolar & $308(35)$ \\
Trimalleolar & $236(27)$ \\
Fracture-dislocation & $334(38)$ \\
Open fracture & $411(47)$ \\
\hline
\end{tabular}

a isolated medial $(\mathrm{n}=23)$ or posterior $(\mathrm{n}=2)$ malleolar fracture, medial malleola fracture associated with posterior malleolus $(n=2)$ or chaput-tillaux fragment $(\mathrm{n}=1)$. 


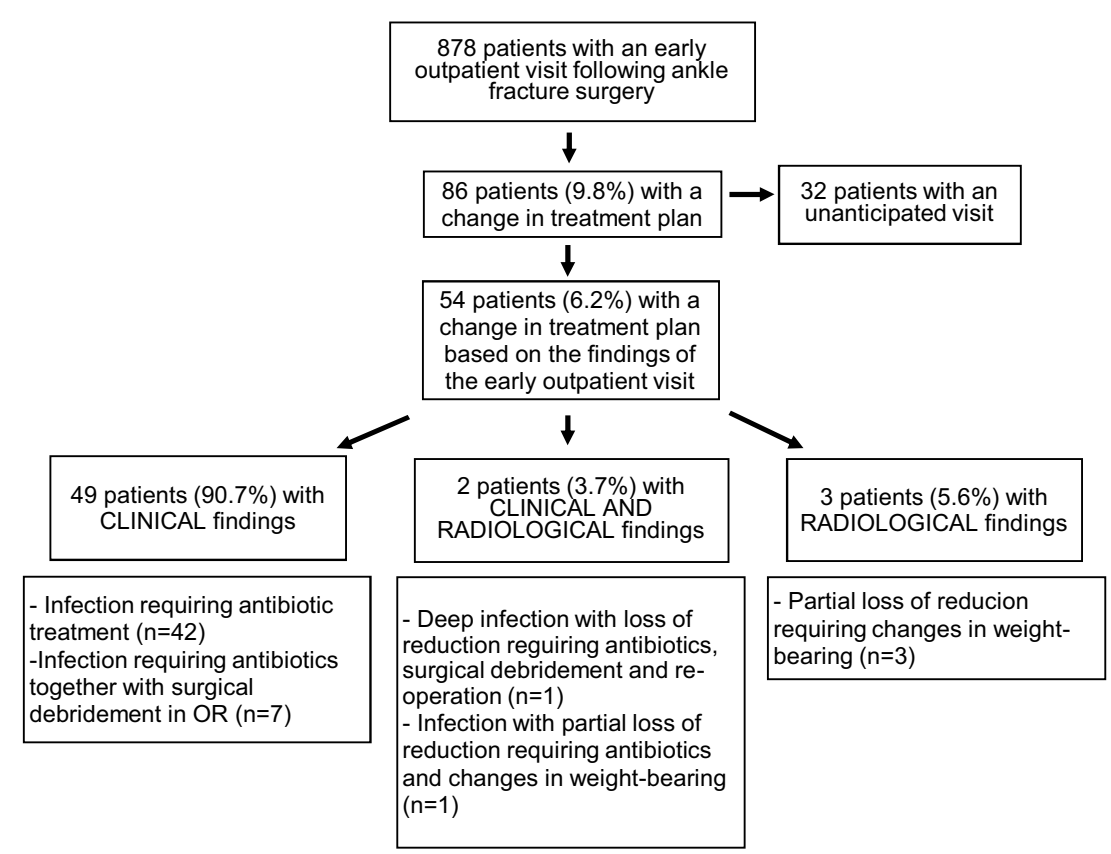

Fig. 1. Flowchart of the included patients showing the causes for a change in treatment plan due to the findings of the first outpatient visit.

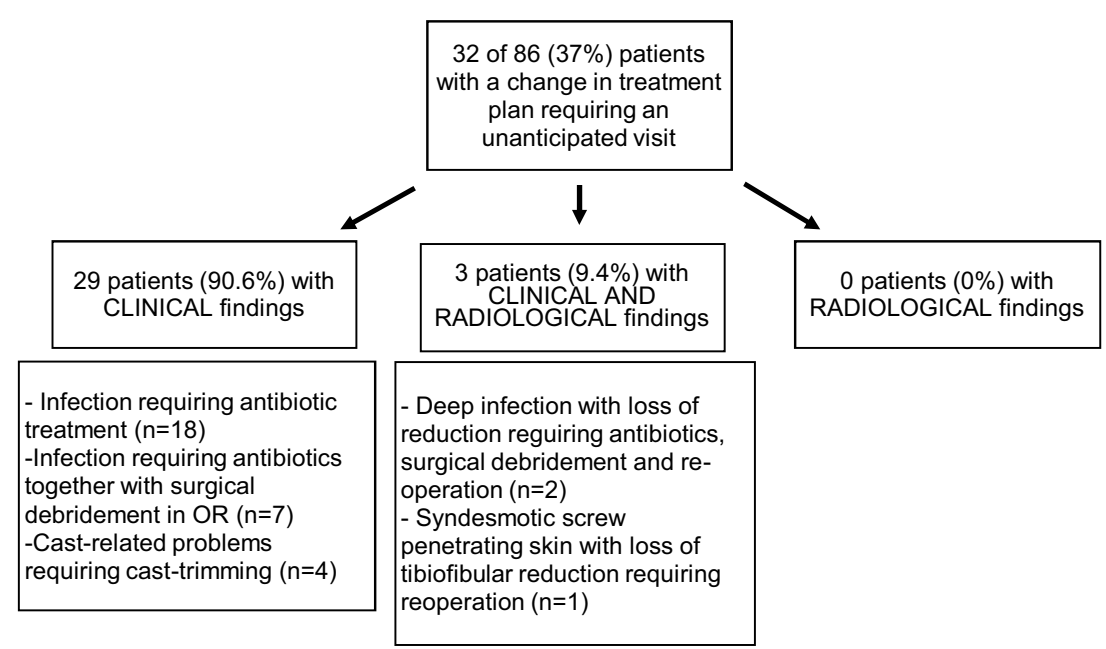

Fig. 2. Flowchart of the patients soliciting an unanticipated control visit prior to the scheduled outpatient visit due to emerging clinical problems related to the ankle fracture surgery.

\section{Discussion}

The present study aimed to assess the impact of the first outpatient visit in a large cohort of ankle fracture patients treated in level-I trauma center. Our results showed that every tenth operatively treated ankle fracture patient requires a change in treatment plan and most changes are exclusively due to clinical findings such as infection or cast-related issues. Unfortunately, only slightly more than one third of the patients with a postoperative clinical problem solicited an unanticipated control before the scheduled outpatient visit. Based on our findings, radiographs should probably not be routinely performed at first outpatient visit following operative treatment in an ankle fracture without clinical signs of a complication.

The incidence of surgical site infection following operative treatment of ankle fractures varies considerably in the literature, ranging from $1.4 \%$ up to $19 \%$ in patients with diabetes [5,8,9-11].
These infections extend total hospital stay and increase healthcare costs by more than $300 \%[12-14]$, and may lead to permanent disability in up to $25 \%$ of patients [15]. Early recognition of these infections is highly important because in cases with delayed diagnosis the presence of biofilm presents a highly demanding challenge for bacterial eradication and infection treatment $[16,17]$. A recent study with 1411 ankle fractures observed that $6.6 \%$ of ankle fracture patients had an early complication [3]. Our study showed that every tenth operatively treated ankle fracture patient required a change in treatment plan by the time of the first outpatient visit, and that $91 \%$ of the changes were exclusively due to clinical findings such as infection or cast-related issues. At the first outpatient visit $8.9 \%$ of all ankle fracture patients presented with signs of infections, however less than one third of these infections were consider as deep at that time point. Since superficial infections may proceed to deep infections, and all patients do not detect these infections themselves, we believe that it important to organize a clinical follow-up visit for these patients 
within the early postoperative period; the stiches or staples are removed and the cast is trimmed if cast-related issues are present. This early outpatient visit would not necessarily require the presence of an orthopaedic surgeon, but instead could be carried out by a health care professional trained to deal with wound healing problems or cast-related issues.

A recent study with operatively treated fractures in heterogenous locations showed that only $0.5 \%$ of patients had a radiographic change from the immediate postoperative radiograph to the outpatient visit radiographs [7]. Our findings with operatively treated ankle fracture patients are in line with these previous findings since our study showed that only three $(0.3 \%)$ patients of cohort of 878 ankle fracture patients required a change in their treatment plan based merely on the findings of the radiographs taken at the outpatient visit. Although the postoperative course was altered in one tenth of operatively treated ankle fracture patients, in the majority of cases the history and the physical examination findings led to the change. Our findings with ankle fractures are very well in line with the previous findings that routine imaging does not provide additional information leading to changes in acute fracture management at the first postoperative visit [7]. Since patients were exposed to unnecessary radiation, we believe that imaging could be delayed to a later follow-up visits in which fracture-healing needs to be assessed. We agree with the previous findings that early postoperative radiographs may not be beneficial in all ankle fracture patients, but radiographs may be indicated if the clinical picture necessitates them [3].

The current study showed, that only $37 \%$ of patients who required a change in their postoperative management had actually solicited an unanticipated visit already before the scheduled control visit due to clinical problems they noted by themselves. Although at hospital discharge patients are given written information on when and where to contact whenever problems emerge, only a little more than one third of the patients were able to understand the severe nature of their clinical symptoms. The study showed that $91 \%$ of patients with an unanticipated visit had sings of an infection or pain due to cast-related problems. Based on our findings, we believe that in addition to the written information given at hospital discharge, all ankle fracture patients should be specifically advised about the possibility of an infection so that they can contact the hospital as soon as possible whenever woundrelated issues occur.

An important limitation of the study is the reliance on data provided by the medical and surgical charts. There is possibility of detection bias since patient data is not always properly documented and some complications such as indolent infections may be underreported. However, significant complications hardly go undetected and we feel confident that we captured all the patients with a complicated outcome. The strengths of this study include the large number of consecutive patients with ankle fractures with early outpatient visit treated at a single institution using a standardized treatment protocol. Furthermore, we consider the large number of treating surgeons increases the generalizability of the results. To our knowledge, this is the most comprehensive analysis of the value of the first outpatient visit following ankle fracture surgery.

The present study showed that only one third of ankle fracture patients are aware of the clinically alarming symptoms and seek care when problems present. Since every tenth operatively treated ankle fracture patient requires a change in the treatment plan due to a clinical problem such as an infection or a cast-related issue, we believe that it is important to control these patients clinically during the early postoperative phase. A prospective randomized study should be carried out to evaluate, whether the outpatient visit could be carried out by a nurse or a paramedic with a readily available consultation from an orthopaedic surgeon whenever alarming symptoms present. Routinely performed radiographs should probably not be obtained at the first outpatient visit in an ankle fracture patient without clinical signs of a complication.

\section{Conflict of interest}

None.

\section{Acknowledgements}

This study was supported by grants from the HUS EVO, the Jane and Aatos Erkko Foundation, the Paulo Foundation, and the Finnish Medical Foundation.

\section{References}

[1] Schepers T, De Vries MR, Van Lieshout EM, Van der Elst M. The timing of ankle fracture surgery and the effect on infectious complications; a case series and systematic review of the literature. Int Orthop 2013;37:489-94.

[2] Somersalo A, Paloneva J, Kautiainen H, Lönnroos E, Heinänen M, Kiviranta I. Incidence of fractures requiring inpatient care. Acta Orthop 2014:85:525-30.

[3] McDonald MR, Bulka CM, Thakore RV, Obremskey WT, Ehrenfeld JM, Jahangir AA, et al. Ankle radiographs in the early postoperative period: do they matter? J Orthop Trauma 2014;28:538-41.

[4] Ovaska M. Complications in ankle fracture surgery. Acta Orthop Suppl 2015;86:1-32.

[5] SooHoo NF, Krenek L, Eagan MJ, Gurbani B, Ko CY, Zingmond DS. Complication rates following open reduction and internal fixation of ankle fractures. J Bone Joint Surg Am 2009;91:1042-9.

[6] Ovaska MT, Mäkinen TJ, Madanat R, Kiljunen V, Lindahl JA. comprehensive analysis of patients with malreduced ankle fractures undergoing re-operation. Int Orthop 2014;38:83-8.

[7] Ghattas TN, Dart BR, Pollock AG, Hinkin S, Pham A, Jones TL. Effect of initial postoperative visit radiographs on treatment plans. J Bone Joint Surg Am 2013;95:e57, doi:http://dx.doi.org/10.2106/JBJS.K.01670.

[8] Ovaska MT, Mäkinen TJ, Madanat R, Huotari K, Vahlberg T, Hirvensalo E, et al. Risk factors for deep surgical site infection following operative treatment of ankle fractures. J Bone Joint Surg Am 2013;95:348-53.

[9] Jones KB, Maiers-Yelden KA, Marsh JL, Zimmerman MB, Estin M, Saltzman CL. Ankle fractures in patients with diabetes mellitus. J Bone Joint Surg $\mathrm{Br}$ 2005;87:489-95.

[10] Schepers T, Van Lieshout EM, De Vries MR, Van der Elst M. Increased rates of wound complications with locking plates in distal fibular fractures. Injury 2011;42:1125-9.

[11] Wukich DK, Joseph A, Ryan M, Ramirez C, Irrgang JJ. Outcomes of ankle fractures in patients with uncomplicated versus complicated diabetes. Foot Ankle Int 2011;32:120-30.

[12] Avilucea FR, Greenberg SE, Grantham WJ, Sathiyakumar V, Thakore RV, Nwosu SK, et al. The costs of operative complications for ankle fractures: a case control study. Adv Orthop 2014;70924:1, doi:http://dx.doi.org/10.1155/2014/709241.

[13] de Lissovoy G, Fraeman K, Hutchis V, Murphy D, Song D, Vaughn BB. Surgical site infection: incidence and impact on hospital utilization and treatment costs. Am J Infect Control 2009;37:387-97.

[14] Regan DK, Manoli 3rd A, Hutzler L, Konda SR, Egol KA. Impact of diabetes mellitus on surgical quality measures after ankle fracture surgery: implications for value-based compensation and pay for performance. J Orthop Trauma 2015;29:e483-486.

[15] Ovaska MT, Mäkinen TJ, Madanat R, Vahlberg T, Hirvensalo E, Lindahl J. Predictors of poor outcomes following deep infection after internal fixation of ankle fractures. Injury 2013;44:1002-6.

[16] Gristina AG, Naylor PT, Myrvik QN. Mechanisms of musculoskeletal sepsis. Orthop Clin North Am 1991;22:363-71.

[17] Trampuz A, Zimmerli W. Diagnosis and treatment of infections associated with fracture-fixation devices. Injury 2006;37:S59-66. 